\title{
Erratum: Developmental Changes in Mental Rotation: A Dissociation Between Object-Based and Egocentric Transformations
}

This erratum reports an error in "Developmental changes in mental rotation: A dissociation between object-based and egocentric transformations" by Sandra Kaltner \& Petra Jansen (Advances in Cognitive Psychology, 12, 67-78. doi: 10.5709/acp-0187-y). The error addresses the fact, that regarding developmental changes in object-based and egocentric transformations, there is only a difference found in children. The incorrect version found changes only in the adult group, but not within children or older adults.

In line with the erratum the following changes need to be considered:

In the abstract, we stated that "Regarding both types of transformations, an advantage of egocentric over object-based human figures was only found in adults which led us tentatively propose that children and older adults show deficits in perspective taking compared to adults." This sentence should be replaced with the following correct information: "Regarding both types of transformations, an advantage of objectbased human figures over egocentric human figures was only found for children, which led us tentatively propose that children show deficits in perspective taking compared to adults and the elderly."

On p. 72 of that article, in the Result section, we stated that „The first interaction of the factors "stimulus" and "group" resulted from the fact that there was a significant difference between the object-based and egocentric human figure condition only within the adult group (BFO: $M=937 \mathrm{~ms}, S D=167 \mathrm{~ms}$; BFE: $M=929 \mathrm{~ms}, S D=141 \mathrm{~ms}$ ), $t(72)$ $=3.58, p=.001$, but not among children (BFO: $M=2032 \mathrm{~ms}, S D=$ $511 \mathrm{~ms}$; BFE: $M=2316 \mathrm{~ms}, S D=714 \mathrm{~ms}), t(59)=-1.62, p=.110$, or older adults (BFO: $M=1687 \mathrm{~ms}, S D=450 \mathrm{~ms}$; BFE: $M=1612 \mathrm{~ms}, S D=$ $459 \mathrm{~ms}), t(61)=1.75, p=.085$, as shown in Figure 2. For a more detailed understanding, all means and standard deviations are given in Table 2. The whole passage needs to be replaced with the following correct information: „The first interaction of the factors "stimulus" and "group" resulted from the fact that there was a significant difference between the object-based and egocentric human figure condition only within the child group (BFO: $M=2032 \mathrm{~ms}, S D=511 \mathrm{~ms}$; BFE: $M=2316 \mathrm{~ms}, S D$ $=714 \mathrm{~ms}$ ), $t(59)=-2.56, p=.013$, but not within the adult group (BFO: $M=937 \mathrm{~ms}, S D=167 \mathrm{~ms} ; \mathrm{BFE}: M=929 \mathrm{~ms}, S D=141 \mathrm{~ms}), t(72)=.38$, $p=.708$, or within older adults (BFO: $M=1687 \mathrm{~ms}, S D=450 \mathrm{~ms}$; BFE: $M=1612 \mathrm{~ms}, S D=459 \mathrm{~ms}), t(61)=.89, p=.383$, as shown in Figure 2. For a more detailed understanding, all means and standard deviations are given in Table 2
Table 2 remains correct. Figure 2 has been corrected.

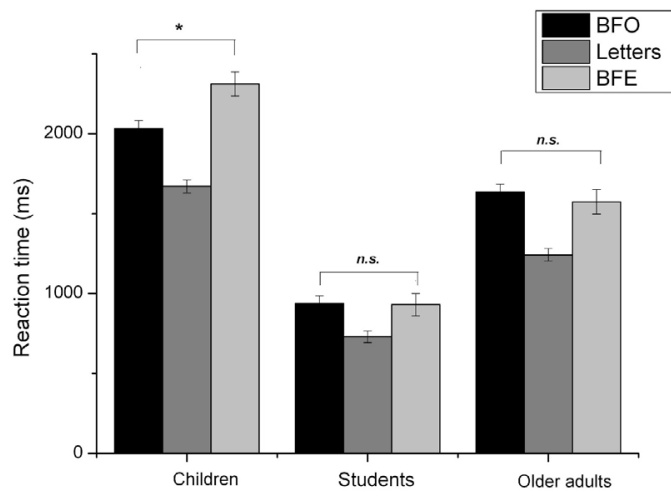

FIGURE 2.

Mean reaction times and standard deviations (error bars) dependent on stimulus type and group. BFO = body figure object, BFE = body figure egocentric task.

In the Discussion section, on p. 72 of that article, we stated that "With respect to the types of transformations, the comparison of RTs in object-based and egocentric transformations revealed that only in adults there was a difference between $\mathrm{BFO}$ and $\mathrm{BFE}$ stimuli expressed by higher RTs for BFO stimuli, which did not occur for both children and older adults. This finding corroborates Hypothesis 3." The whole passage needs to be replaced with the following correct information: "With respect to the types of transformations, the comparison of RTs in object-based and egocentric transformations revealed that only in children there was a difference between BFO and BFE stimuli expressed by higher RTs for BFE stimuli, which did not occur for both students and older adults. This finding is not on line with Hypothesis 3."

Corresponding author: Sandra Kaltner, University of Regensburg, Universitätsstraße 31, 93053 Regensburg, Phone: +49 941507 5131. E-mail: sandra.kaltner@ur.de 
On p. 74, we stated that "Analyses showed that whereas RTs did not differ between object-based and egocentric human figures in children and older adults, adults needed longer to solve BFO stimuli compared to BFE human figures. The performance advantage of egocentric transformations over object-based rotations in the adults group of the present study is in line with previous literature (Amorim \& Stucchi, 1997; Creem et al., 2001; Wraga et al., 2000, 2005). However, this performance advantage of egocentric transformations could not be revealed for children and older adults. We tentatively propose that the absence of an egocentric advantage in children and older adults could be interpreted as decreased performance restricted to this kind of transformation. Besides, it supports the findings of older adults provided by several studies (Devlin \& Wilson, 2010; Jansen \& Kaltner, 2014)." The whole passage needs to be replaced with the following correct information: "Analyzes showed that whereas reaction times between object-based and egocentric human figures did not differ in adults and older adults, children needed longer to solve BFE-stimuli compared to BFO-human figures. That is, they showed an impaired performance in egocentric transformations, which is not present in either adults or older adults. Thus, whereas children seem to be limited in the egocentric transformation, this disadvantage seems to diminish with increasing age. However, it still remains open why the advantage of egocentric transformations found in adults (Amorim \& Stucchi, 1997; Creem et al., 2001, 2002; Wraga et al., 2005) was not replicated in the present study. We tentatively account the stimulus material as contributing factor for the observed results. According to Amorim, Isableu and Jarraya (2006), the embodied stimulus material (e.g. adding body characteristics on abstract cubes) facilitates the mapping of the own body axes onto the stimulus resulting in an increased performance compared to the use of abstract cube figures. The diminishing advantage of egocentric over object-based transformations in adults and older adults of the present study would argue for the idea that objectbased rotations seem to benefit from embodying stimulus material. This is in line with the results of Kaltner, Jansen and Riecke (2017), who also showed that object-based rotations benefited from stronger embodiment effects of life-sized stimuli compared to small, artificial ones presented on the computer screen. Therefore, a closer examination of the meaning of the stimulus material in inducing embodiment effects with focus on developmental changes could be an interesting topic for future research."

On p. 75, we stated that "Devlin and Wilson (2010) claimed that the decline in an egocentric transformation task might be due to the difficulty of integrating information relevant for the body schema. The body schema integrates "information about the position and extent of the human body (...) and therefore represents a spatiomotor representation of the body" (p.182, Buxbaum, Giovannetti, \& Libon, 2000). In older adults, it was shown that the noise of neuronal signals from sensorimotor areas (e.g., posterior parietal cortex) increases with age which leads to a decreased ability to integrate information in order to build a stable representation of the own body (Ghafouri \& Lestienne, 2000)." The whole passage refers to incorrect results and should be ignored.
In the Limitations and Conclusions section, on p. 75, we stated that: "In summary, this study revealed two important findings: 1) the role of an age-related decline in processing speed and the possible importance of WM capacity in MR performance; 2) the observation that children and older adults seem to show deficits in perspective taking compared to adults. This finding supports previous work (Devlin \& Wilson, 2010; Piaget \& Inhelder, 1971) but sticks out by using a standardized design for each age group assessed. Therefore, we tentatively propose that perspective transformations are more sensitive to developmental change compared to object-based transformations." The whole passage needs to be replaced with the following correct information: "In summary, this study revealed two important findings: 1) the role of an age-related decline in processing speed and with the possible importance of WM capacity in MR performance; 2) the observation that only children seem to show deficits in perspective taking compared to adults and older adults. This finding supports the previous work (Piaget \& Inhelder, 1956; Piaget \& Inhelder, 1971), but sticks out by using a standardized design for each age group assessed. Therefore, we tentatively propose, that perspective transformations are only in childhood impaired compared to object-based transformations. This leads to the remaining question, when perspective transformations are exactly required. This study provides a first step to investigate this issue, but should be specified by future research."

The following References must be added based on the correct result:

Amorim, M. A., Isableu, B., \& Jarraya, M. (2006). Embodied spatial transformations: "Body analogy" for the mental rotation of objects. Journal of Experimental Psychology: General, 135, 327-347. doi: 10.1037/0096-3445.135.3.327

Kaltner, S., Jansen, P., \& Riecke, B. E. (2017). Stimulus size matters: Do life-sized stimuli induce stronger embodiment effects in mental rotation? Journal of Cognitive Psychology, 29, 1-16. doi: 10.1080/20445911.2017.1310108

References based on the incorrect version which should be ignored:

Buxbaum, C. J., Giovanetti, T., \& Libon, D. (2000). The role of the dynamic body schema in praxis: Evidence from primary progressive apraxia. Brain, 44, 166-191. doi: 10.1006/brcg.2000.1227

Ghafouri, M., \& Lestienne, F. G. (2000). Altered representation of peripersonal space in the elderly human subject: A sensorimotor approach. Neuroscience Letters, 289, 193-196. doi: 10.1016/ S0304-3940(00)01280-5 